\title{
Philosophy and Feasibility of Dual Readout Calorimetry
}

The main simple idea is to measure a hadronic shower twice - as if two different calorimeters make an image of the same shower.

The first realization of this was the Dual Readout Module (DREAM) embedded with scintillating fibers and clear fibers (for Cerenkov light production) described by Wigmans, $7^{\text {th }}$ Calorimetry Conf., Tucson, 1997.

Scintillation light, S: all charged particles, mostly $\pi^{ \pm}$, some slow $\mathrm{p}$

Cerenkov light, $\mathrm{C}$ : $\quad$ charged particles above $\beta>1 / n$, mostly $\mathrm{e}^{ \pm}$from $\pi^{0} \rightarrow \gamma$

The main goal was to improve hadronic calorimetry without the constraint that the sensitive-medium-to-absorber ratio be compensating, $e / h=1$. 


\section{A few "applications"}

1. DREAM-1 (Scintillation+Cerenkov, exact separation of light in separate fibers)

2. DREAM-2 (Scintillation+Cerenkov light mixed into the same fibers)

3. DREAM-3, $\mathrm{PbWO}_{4}$ (Scintillation+Cerenkov in one crystal: 4th concept, CMS-2)

4. $\mathrm{TeV} \gamma$-ray astroparticle physics (Cerenkov $+\mathrm{N}_{2}$ fluorescence)

5. Muon identification (4th)

\section{(There must be a half-dozen more ...)}


This dual measurement doesn't have to be just with light...

- Dense spatial clusters: weight "EM" clusters

$\square \square$ [Abramowicz, et al., NIM 180 (1981) 429]

- "two samplers": nice early discussion of possibilities

[Paul Mockett, Calorimeter Review, SLAC Report 267, 1983]

- $d E / d x$ \& Cerenkov: in L-Argon calorimeter

[Winn and Worstell, IEEE NS-36 (1989) 334]

- Radial Scintillation \& Cerenkov profiles

$$
\text { [ DREAM "profile" paper, NIM A548 (2005) 336] }
$$




\section{Mockett 1983 SLAC Summer Institute}

- "A technique is needed that is sensitive to the relative fraction of electromagnetic energy and hadronic energy deposited by the shower. This could be done hypothetically if the energy were sampled by two media: one which was sensitive to the beta equals one electrons and another which was sensitive to both the electrons and other charged particles. For example one sampler could be lucite which is sensitive only to the fast particles, while the other sampler could be scintillator. Then the fraction of pizeros produced could be determined from the relative pulse heights of the two samplers. Another technique might be to utilize the slow scintillation pulse and the fast Cerenkov pulse in total absorbing materials such as scintillating glass or Barium fluoride. By appropriate gating for wave form sampling ..." 
This dual measurement doesn't have to be just with separate fibers, or "samplers"

- Mixed light in one set of fibers ( DREAM-2)

- Mixed light in a single crystal (4th, CMS-2, DREAM-3 )

- Mixed light in the atmosphere ( TeV astrophysics )

- Mixed light in noble liquids ... 


\section{But, you must separate the light ... \\ Cerenkov \\ Scintillation}

- Physically, e.g., separate fibers clear fiber fiber with fluor

- By direction:

$\theta_{\text {Cerenkov }} \sim 45^{0}$ isotropic

- By wavelength: $\mathrm{d} \lambda / \lambda^{2} \sim$ blue, $\mathrm{UV}$ green, etc.

- By time:

- By polarization few $\%($ avg)

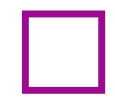
none 


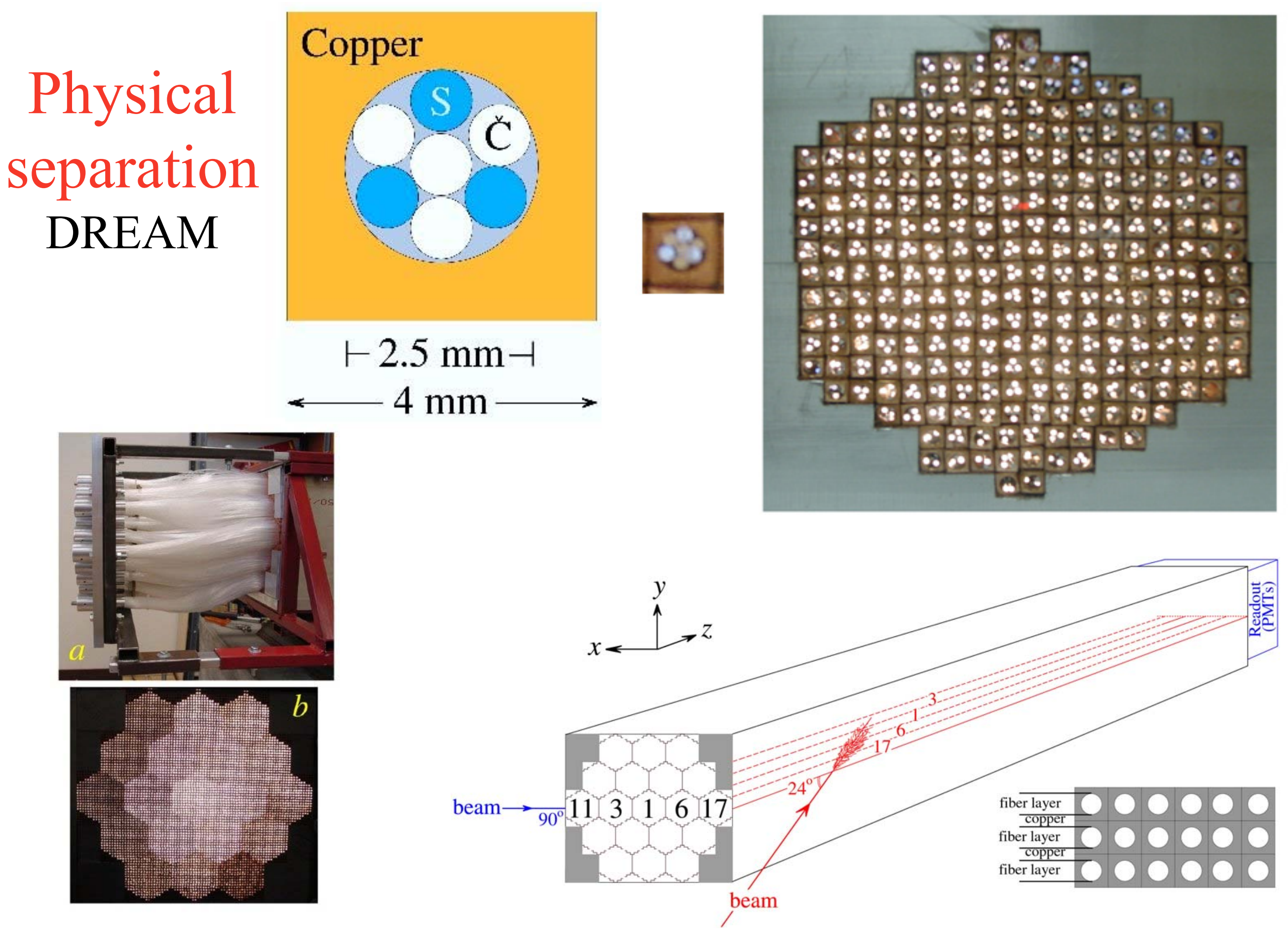




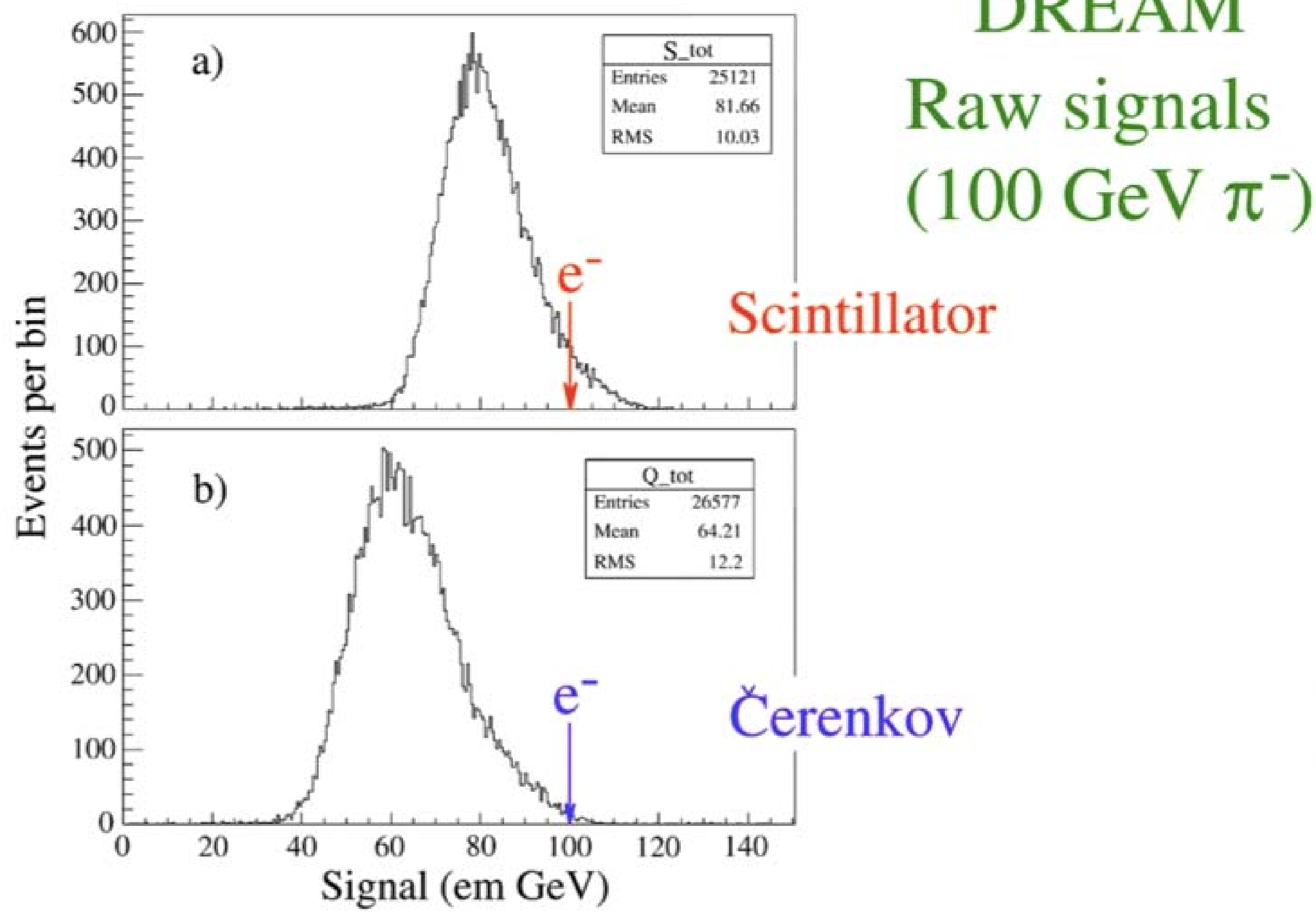




\section{DREAM: Cerenkov vs. scintillation signal $100 \mathrm{GeV} \pi^{-}$}

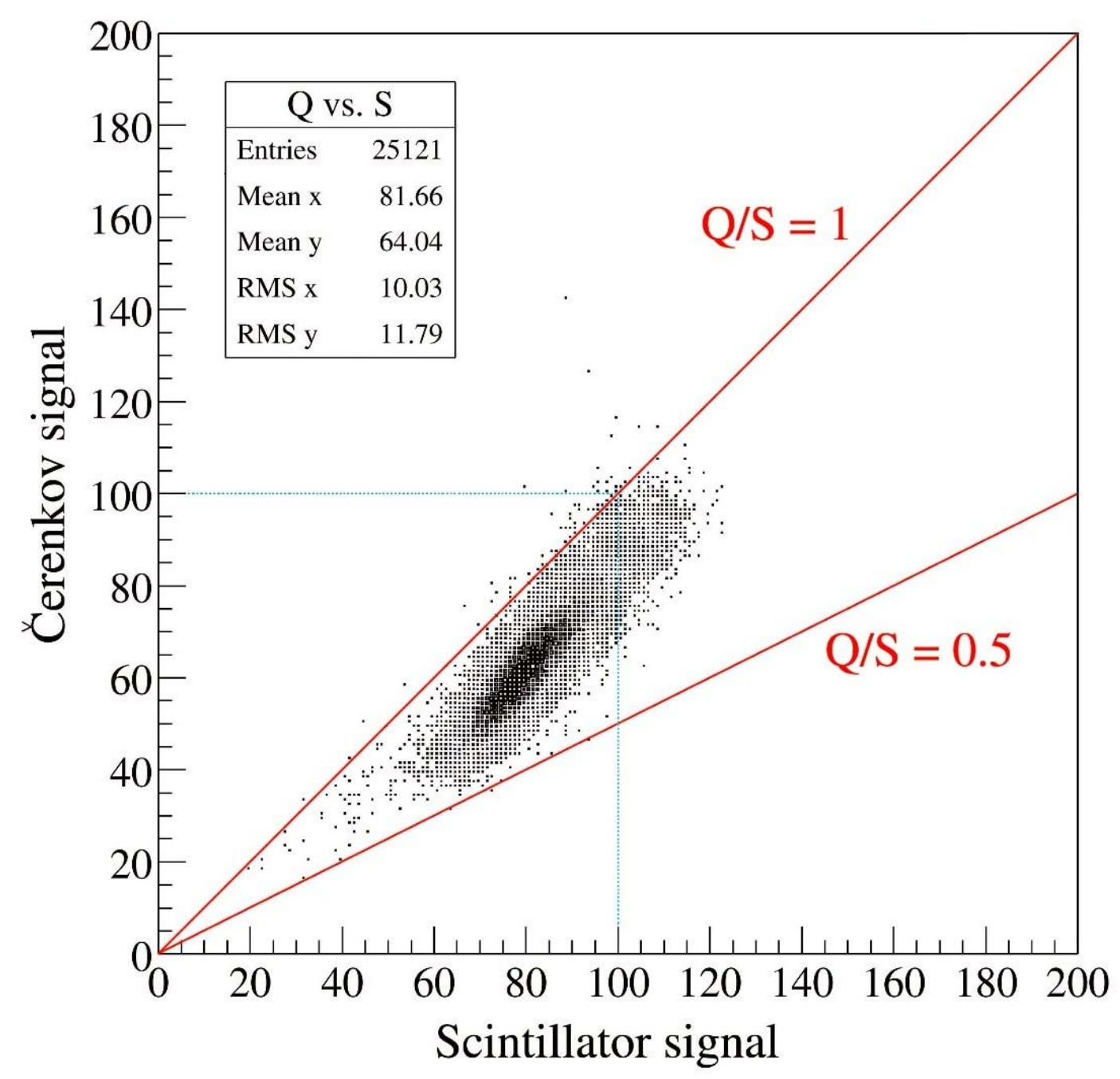

Raw data for $100 \mathrm{GeV}$

$\pi$ - Cerenkov vs

Scintillation signals,

scale defined by response to $40 \mathrm{GeV} e^{-}$.

EM fraction

fluctuations are obvious

June 2006

J. Hauptman CALOR06 Chicago 
Cerenkov signal is well correlated with EM fraction

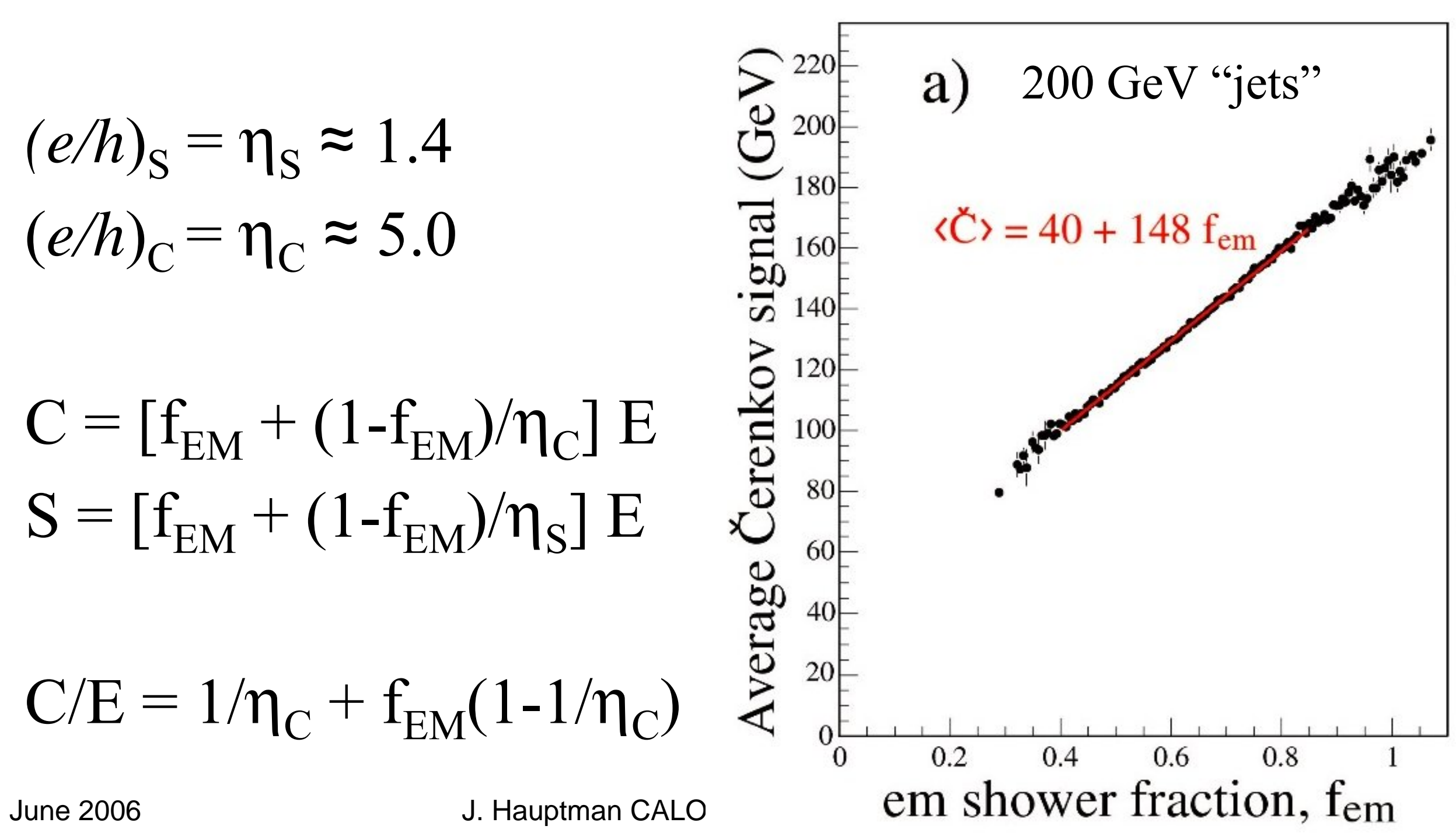




\title{
DREAM data $200 \mathrm{GeV} \pi$ : Energy response
}

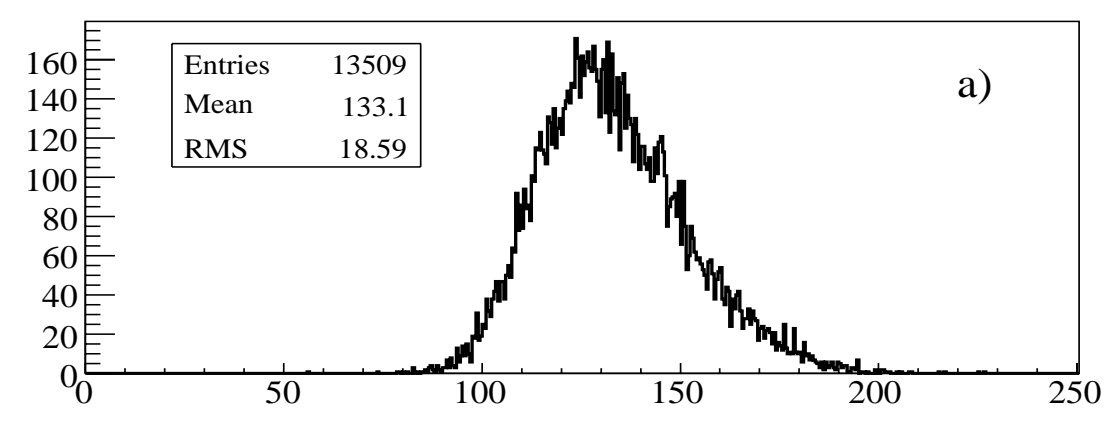

\author{
Scintillating fibers
}

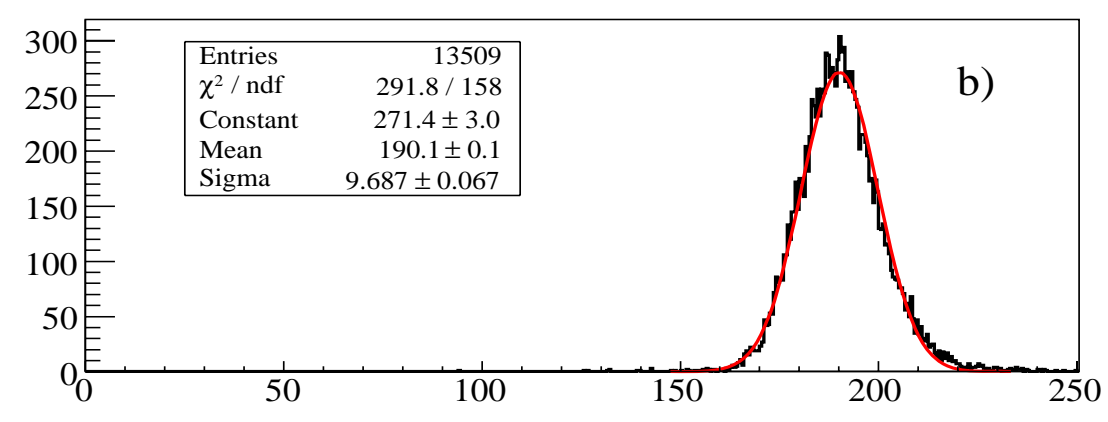

$$
\text { Scint }+ \text { Cerenkov }
$$

$\mathrm{f}_{\mathrm{EM}} \propto\left(\mathrm{C} / \mathrm{E}_{\text {shower }}-1 / \eta_{\mathrm{C}}\right)$

(4\% leakage fluctuations)

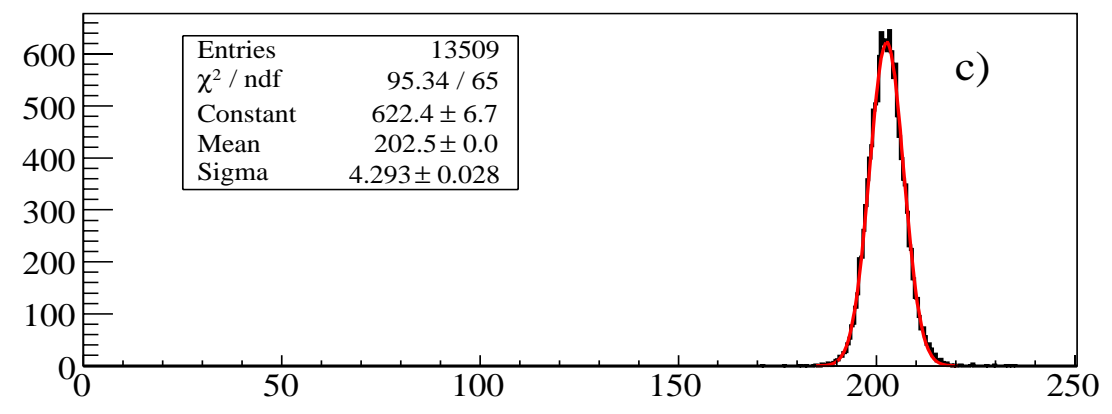

Scint + Cerenkov

$$
\mathrm{f}_{\mathrm{EM}} \propto\left(\mathrm{C} / \mathrm{E}_{\text {beam }}-1 / \eta_{\mathrm{C}}\right)
$$

(suppresses leakage)

June 2006

Data NIM A537 (2005) 537.

J. Hauptman CALOR06 Chicago 


\section{More important than good Gaussian response: DREAM module}

calibrated with $40 \mathrm{GeV}$ e- into the centers of each tower responds linearly to $\pi^{-}$and "jets" from 20 to $300 \mathrm{GeV}$.

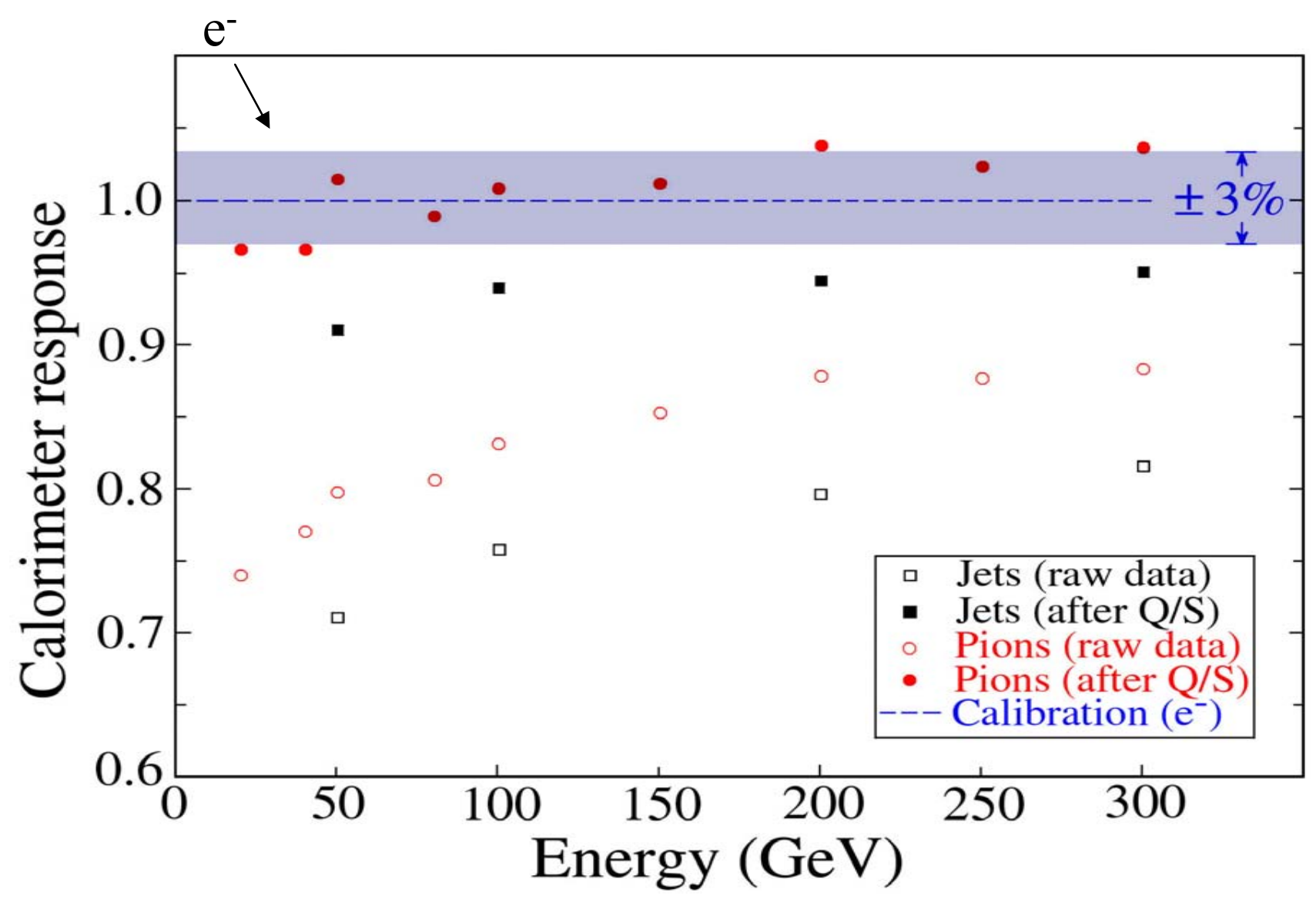

Hadronic linearity may be the most important achievement of dualreadout calorimetry, so far. 


\section{Deliberately mixing the light}

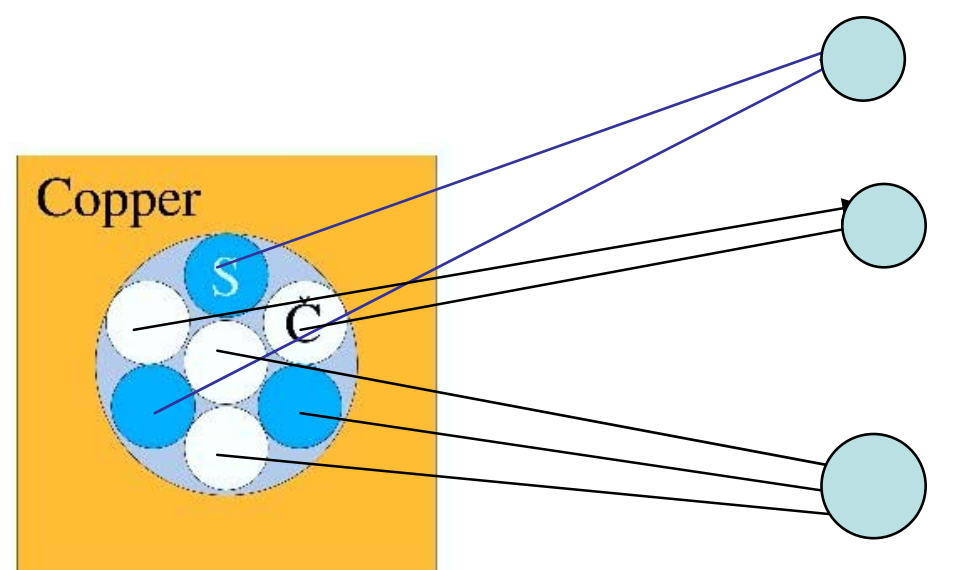

$\mathrm{S}+\mathrm{S}=$ pure scintillation

$\mathrm{C}+\mathrm{C}=$ pure Cerenkov

$\mathrm{S}+\mathrm{C}+\mathrm{C}=$ mixed light

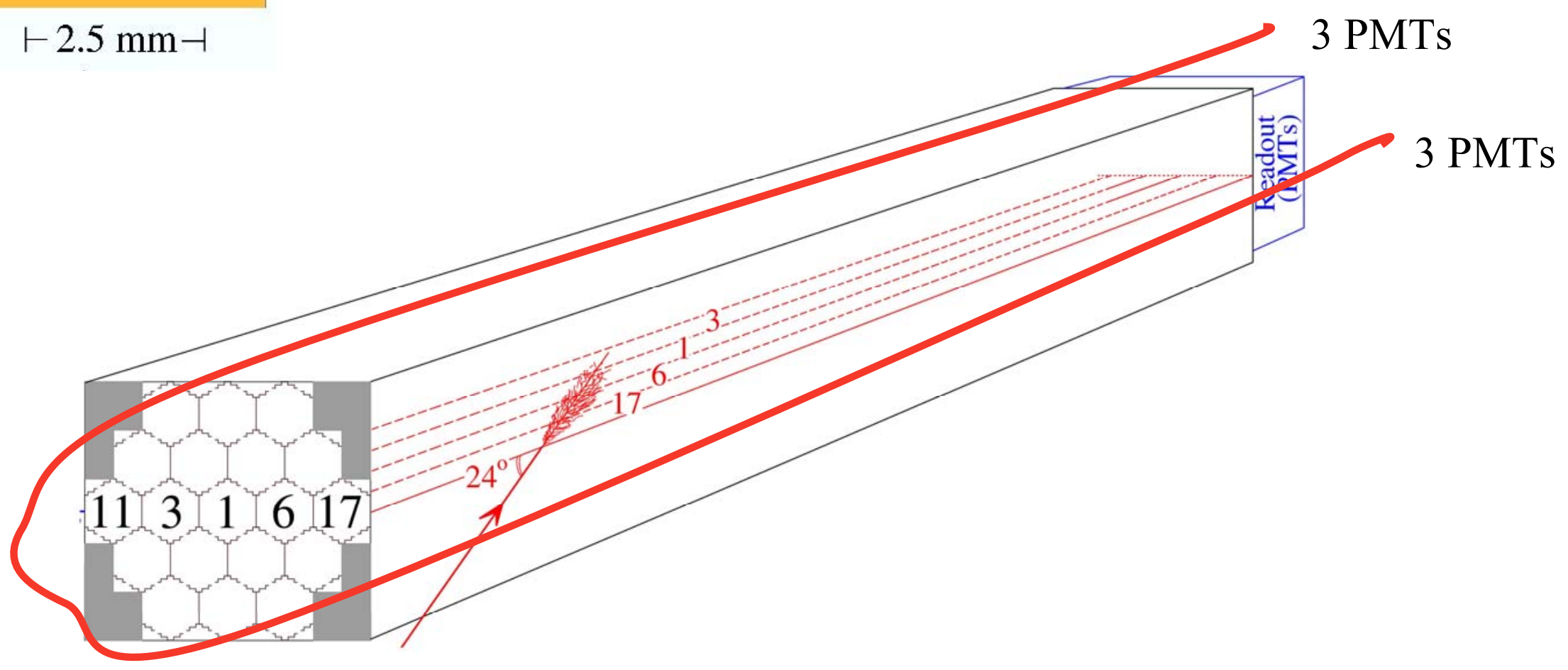




\section{Separation by direction: mixed light}

DREAM-2 test, scintillation and

Cerenkov light deliberately mixed in same fibers

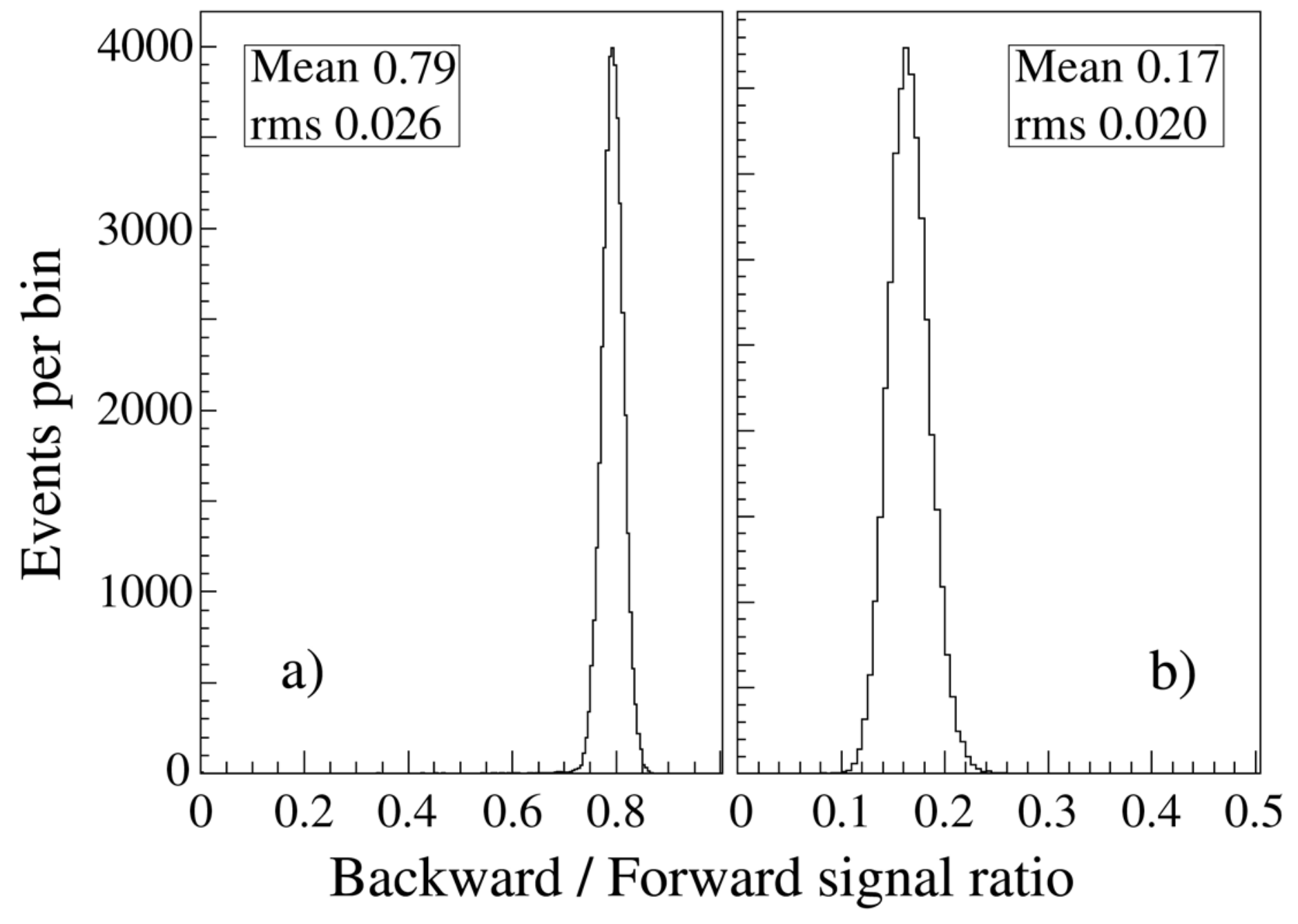


Time separation: DREAM-2 module, mixed light in fibers

Most of the light is inside 10-15 ns. A deliberate design would improve this separation. These signals were digitized at the ends of long cables.

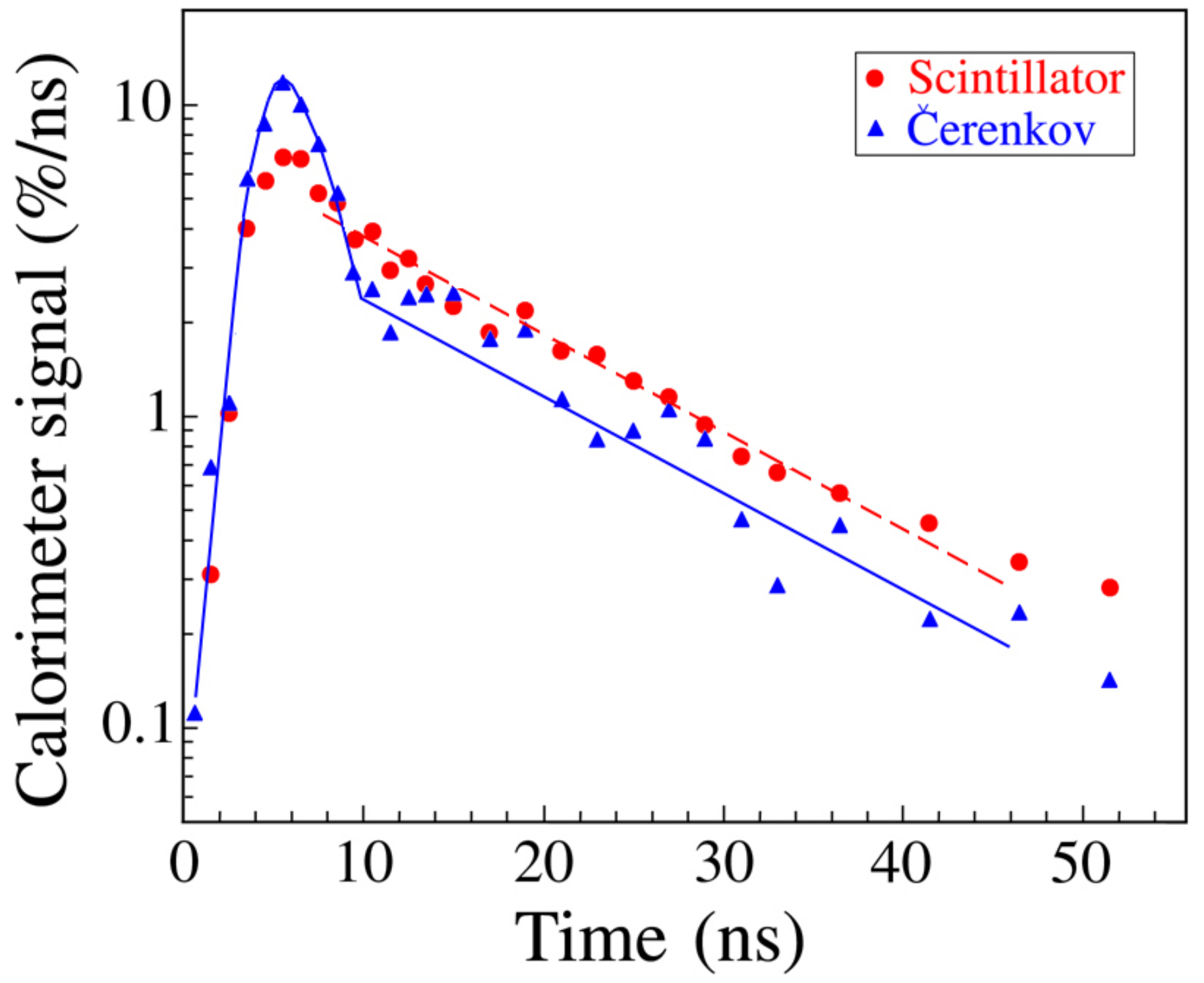


Light mixed in a single macroscopic medium

\section{Signal distributions $\mathrm{PbWO}_{4}$ crystal (cosmics)}

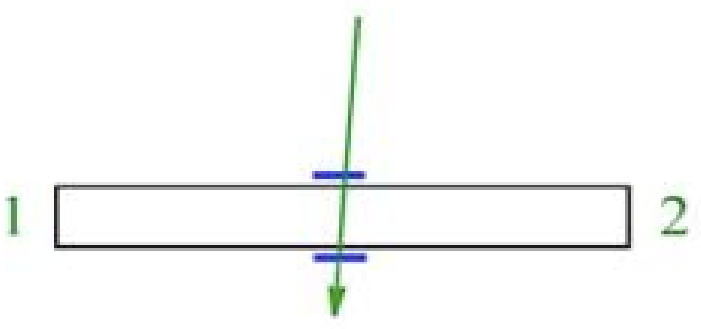

Average energy deposit (mip):

$9.4 \mathrm{MeV} / \mathrm{cm} \longrightarrow 23.5 \mathrm{MeV}$

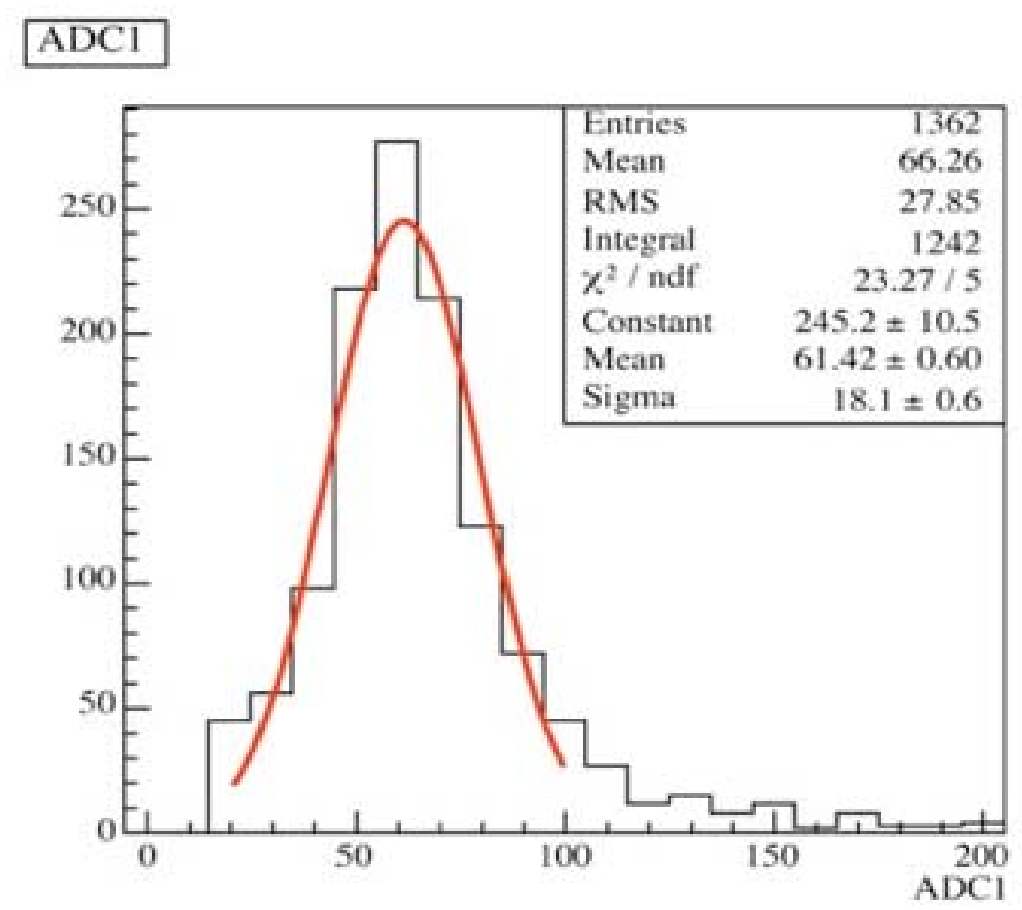

June 2006

J. Hauptman CALOR06 Chicago

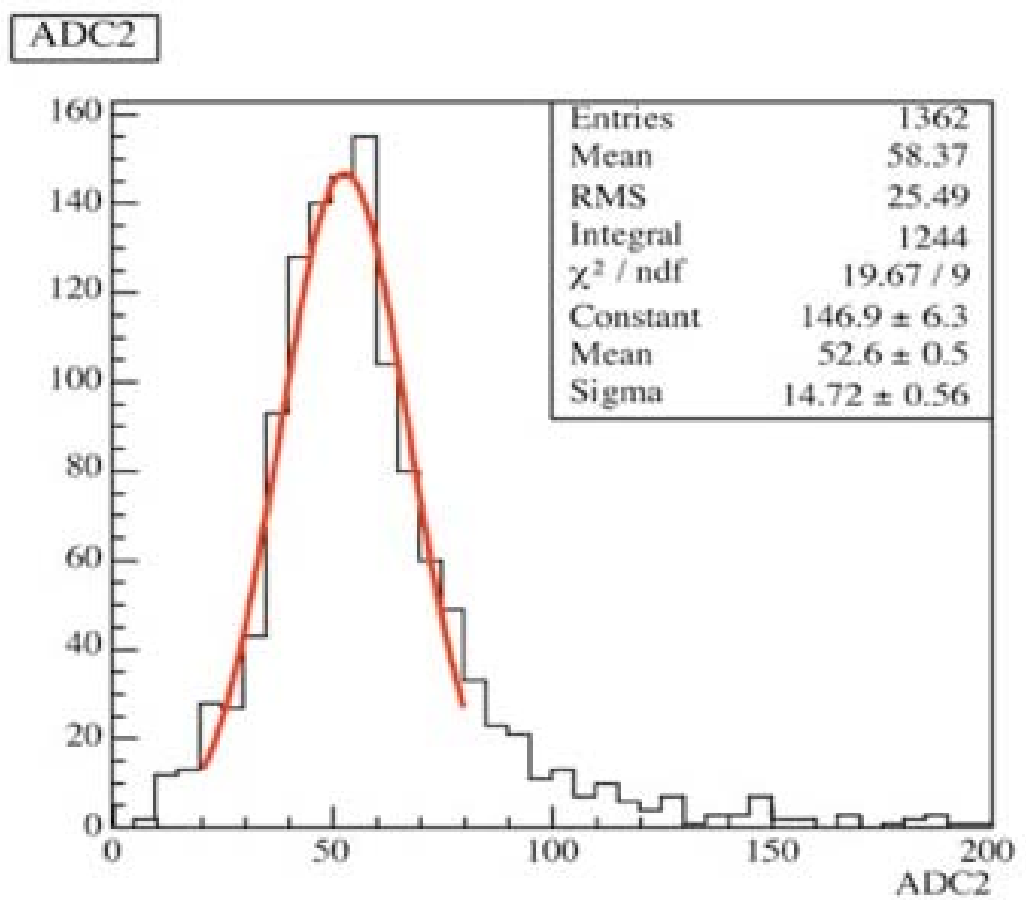

R.Wigmans Elba '06 


\section{Identifying Cerenkov component on the basis of its directionality}

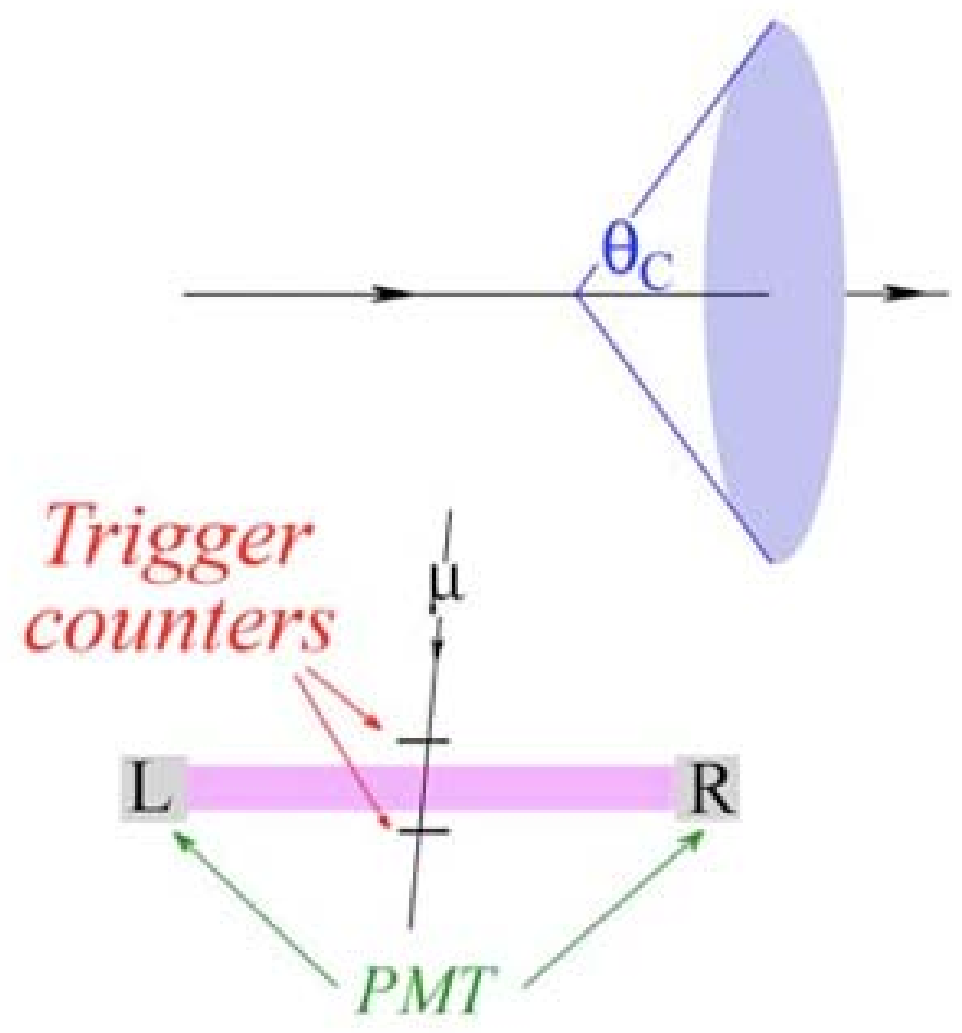

$$
n=2.2, \arccos \theta_{C}=1 / n \rightarrow \theta_{C}=63^{\circ} .
$$

Calibration:
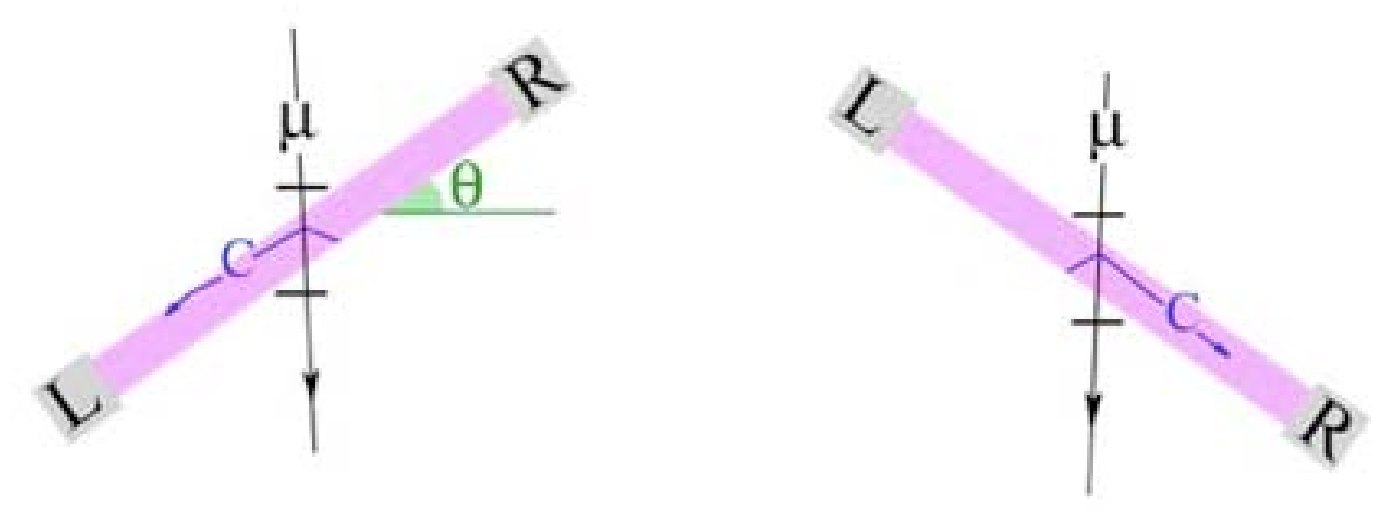

$$
\mathrm{L}=\mathrm{R}
$$

$$
\mathrm{L}>\mathrm{R} \quad \mathrm{L}<\mathrm{R}
$$

$\rightarrow$ Measure $\frac{\mathrm{L}-\mathrm{R}}{\mathrm{L}+\mathrm{R}}$ as a function of $\theta_{\mathrm{R} \text {. Wigmans Elba “06 }}$ 
Experimental results, light directionality in $\mathrm{PbWO}_{4}$

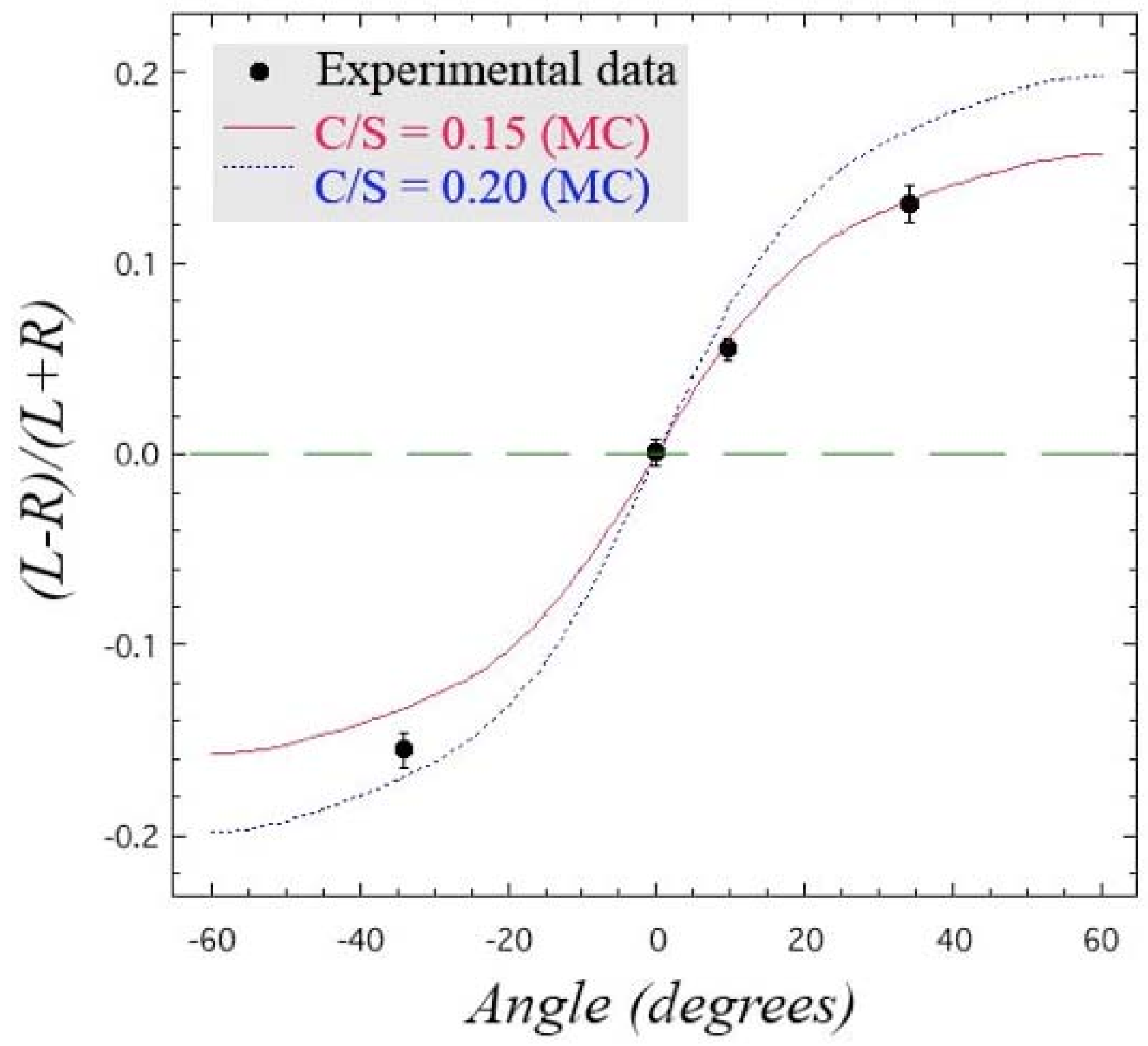


Iime structure of cosmic ray events in $\mathrm{PbWU}_{4}(\Delta \mathrm{E}=2 \mathrm{MeV})$
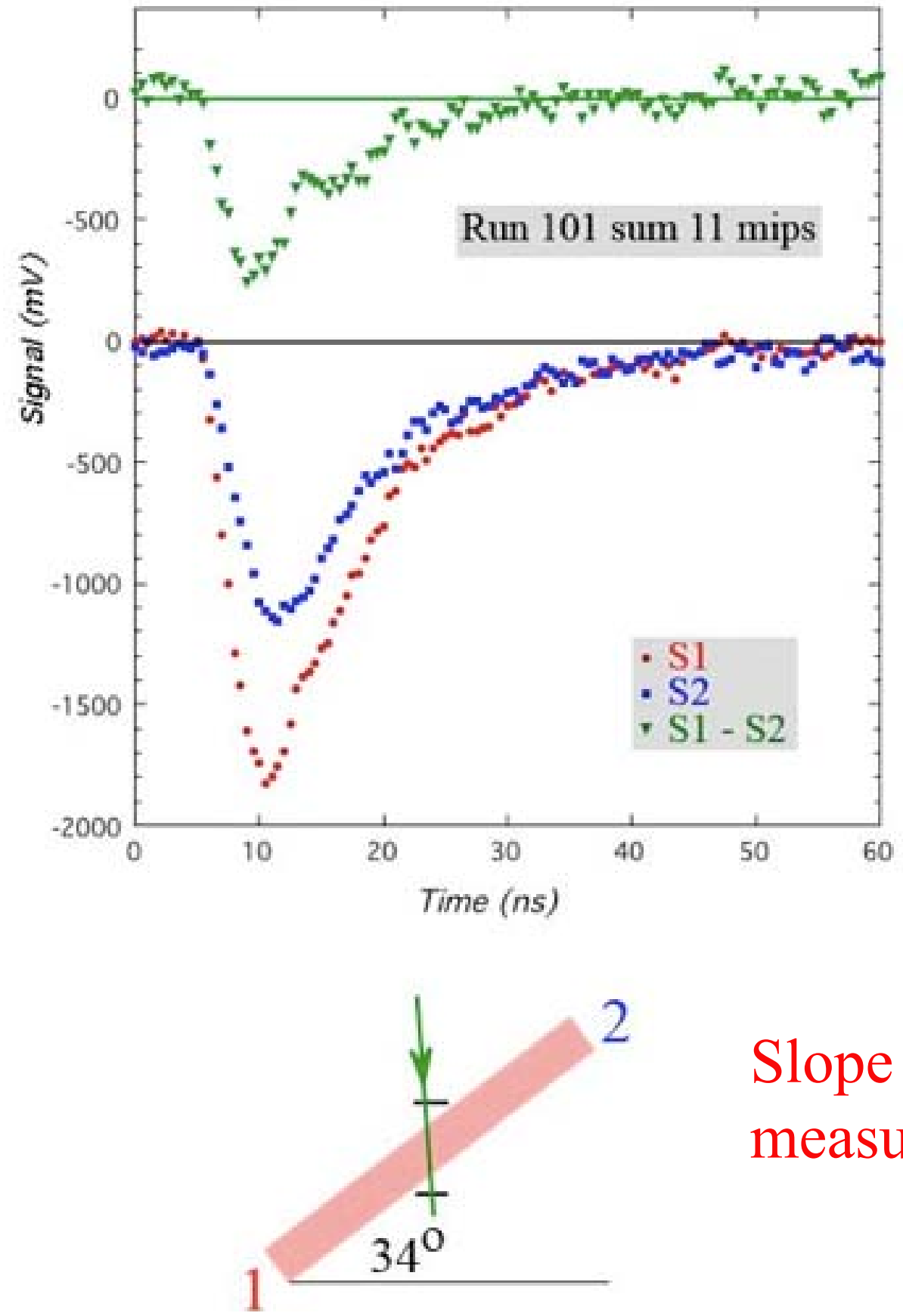

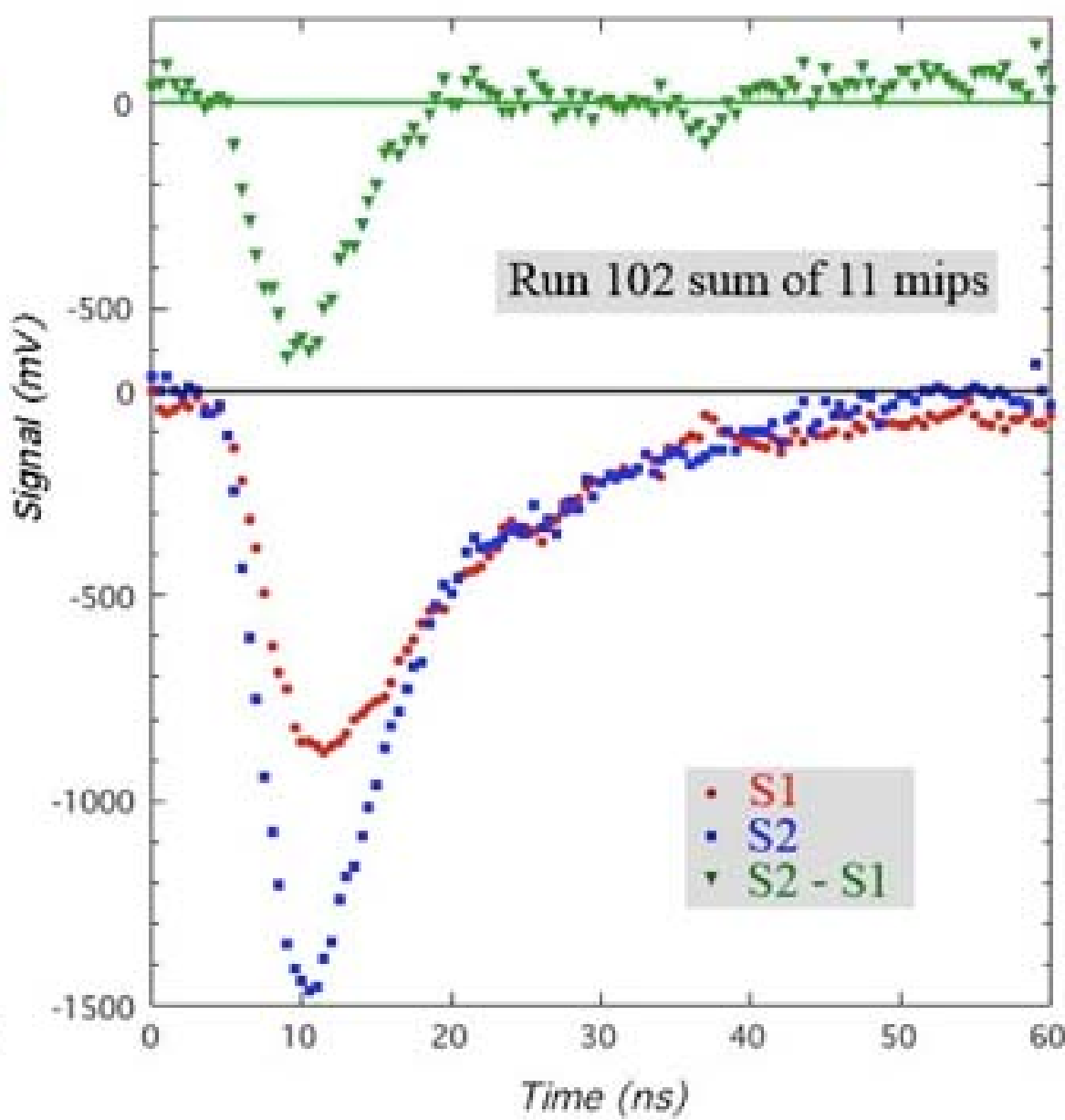

Slope $d L / d t$ measures $\mathrm{C} / \mathrm{S}$

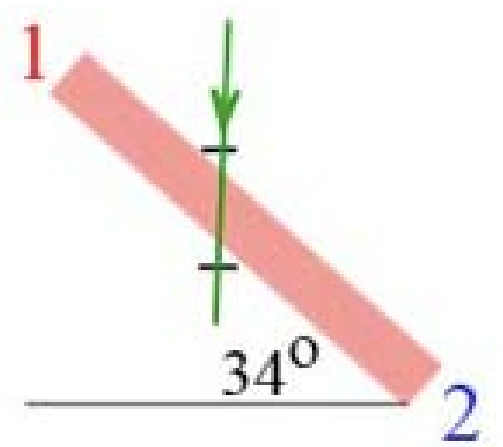


Time structure of cosmic ray events in $\mathrm{PbWO}_{4}$ (Landau tail)
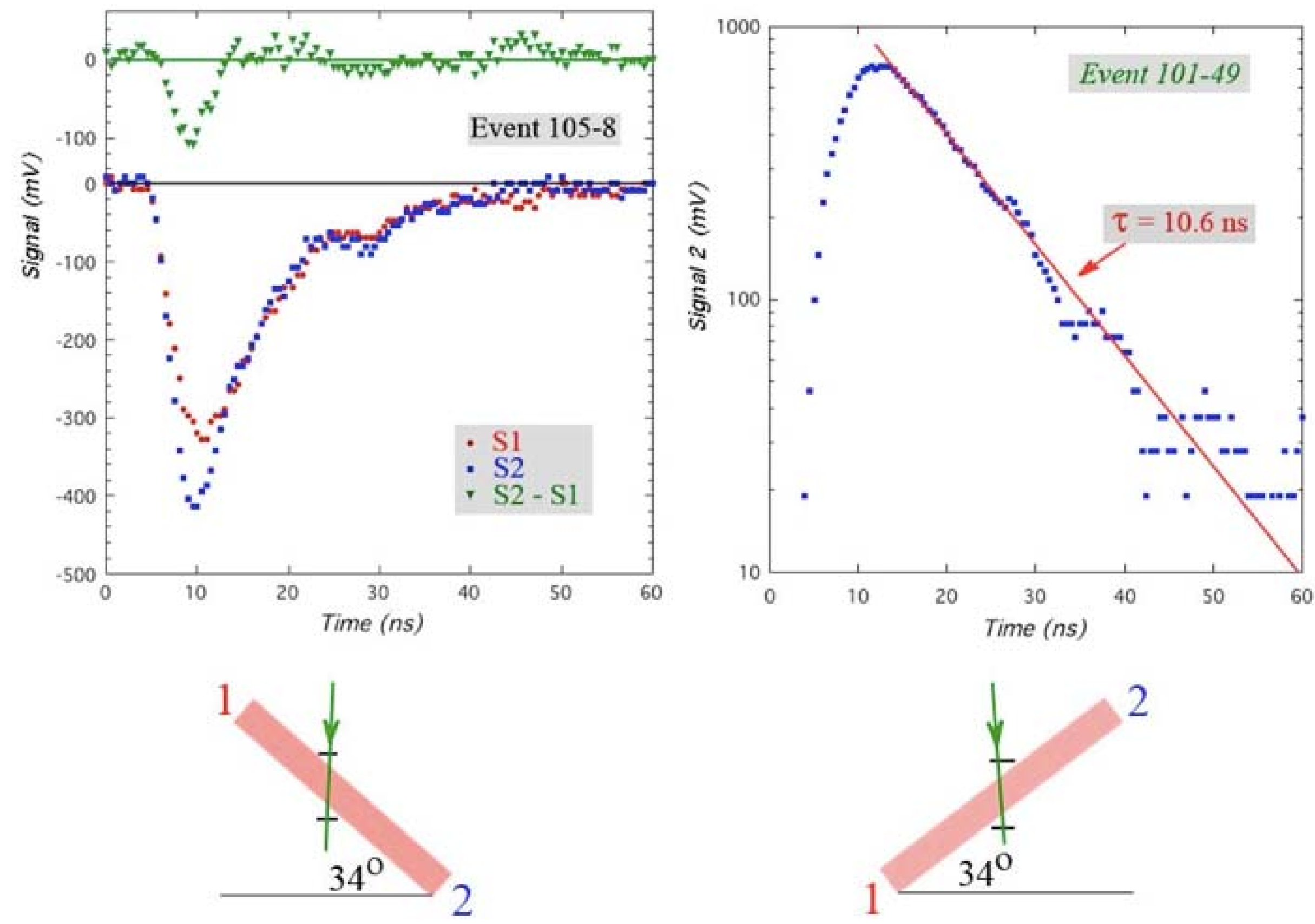


\section{"Lamborghini" model crystal (CMS-2 ?)}

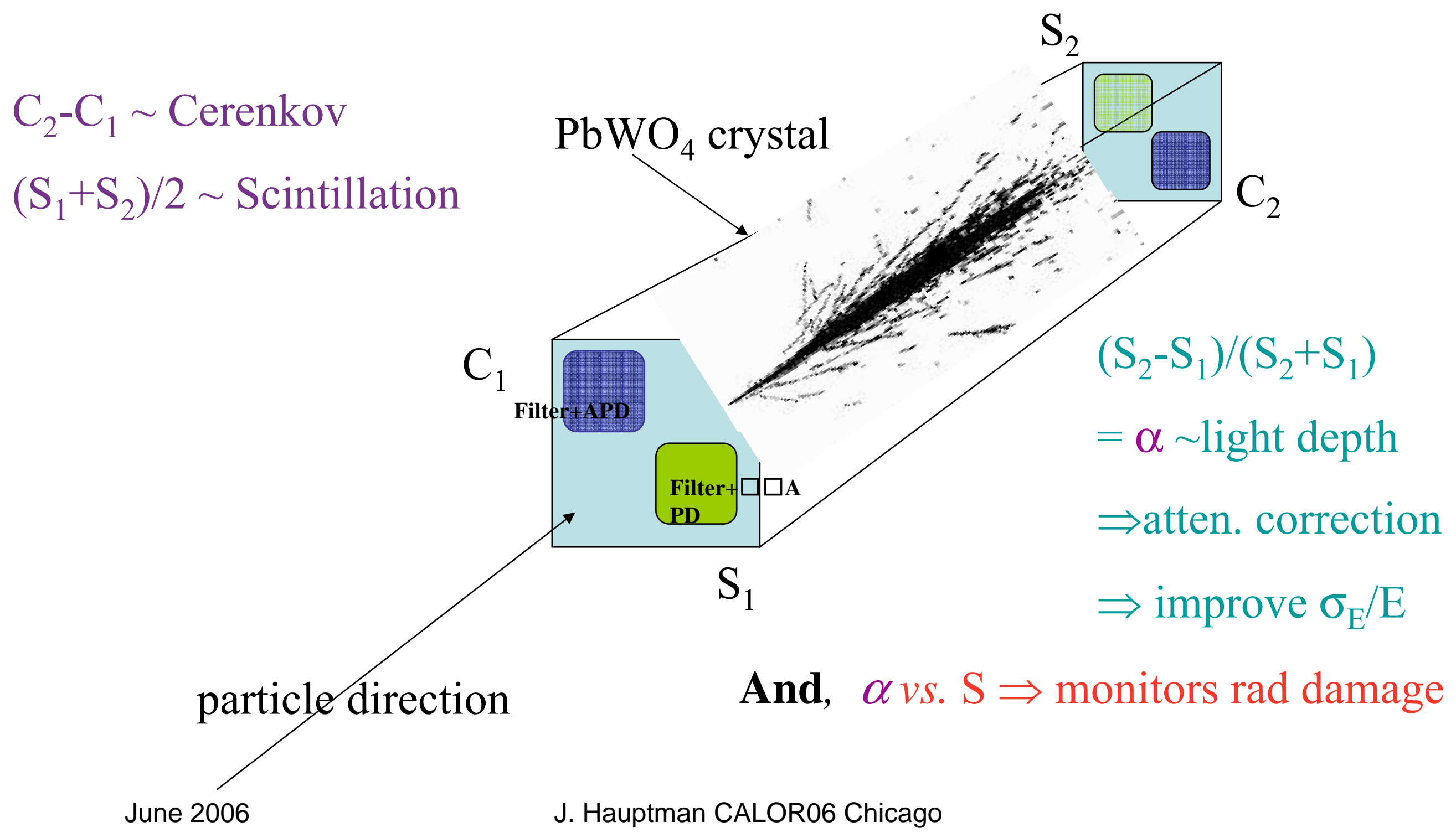


$\mathrm{TeV}$ particle astrophysics: separate $\gamma$ from proton, $\mathrm{He}$, and more ... problem at the Greisen (GZK) limit ?

- Index of air $n=1+.00029 \rho_{\mathrm{NTP}}$

- Cerenkov $\beta_{\mathrm{th}}=1 / n$

$\mathrm{N}_{2}$ line emissions $300-400 \mathrm{~nm}$ and with lifetimes of several ns
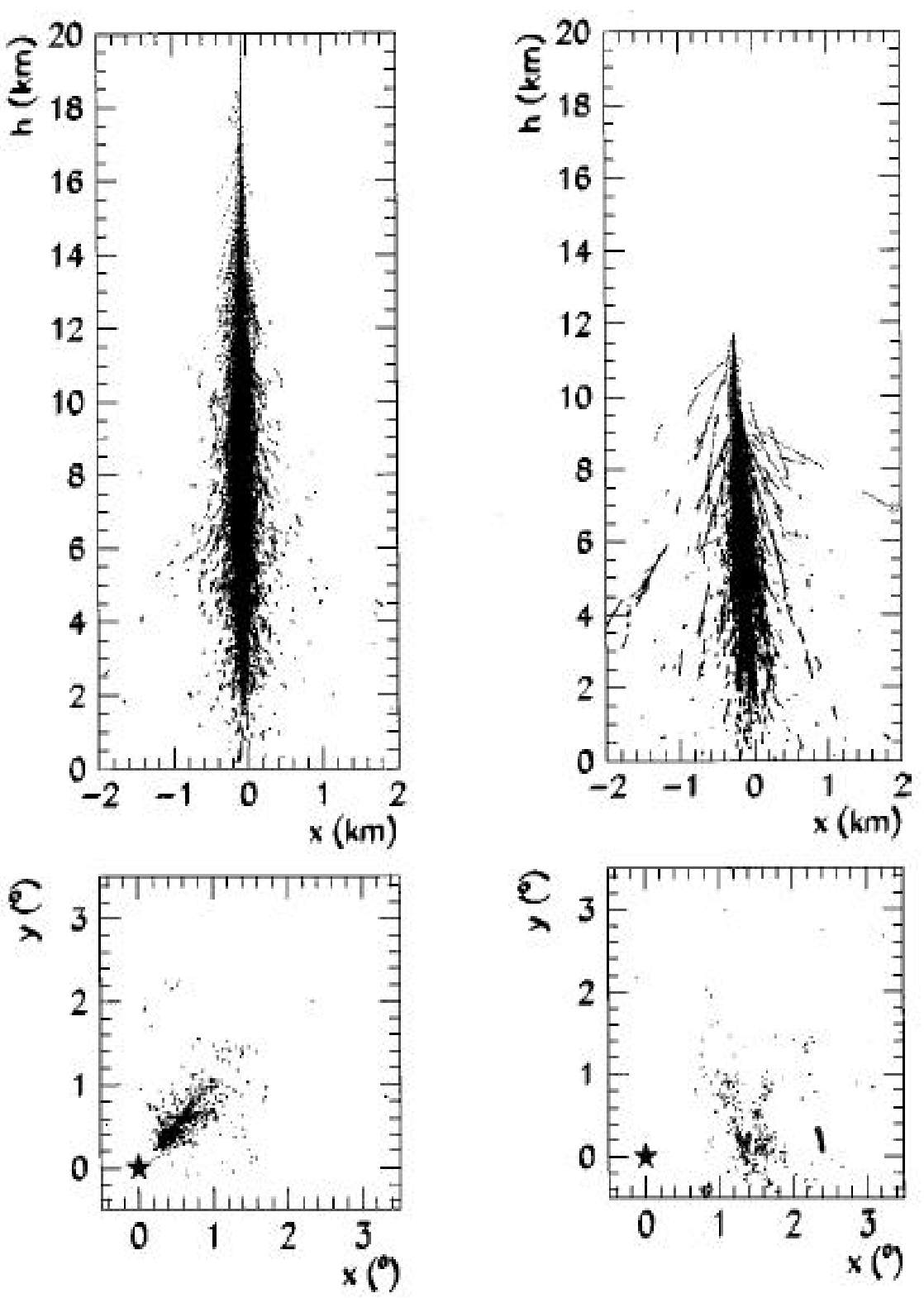

June 2006

$\gamma$-ray

J. Hauptman CALOR06 Chicago proton 
Hadronic showers in the atmosphere: separate by time and wavelength

Scintillation light for $\mathrm{N}_{2}$ fluorescence ... (green and slow)

Pancake of Cerenkov light ( $\sim$ m thick)

... (blue and fast)

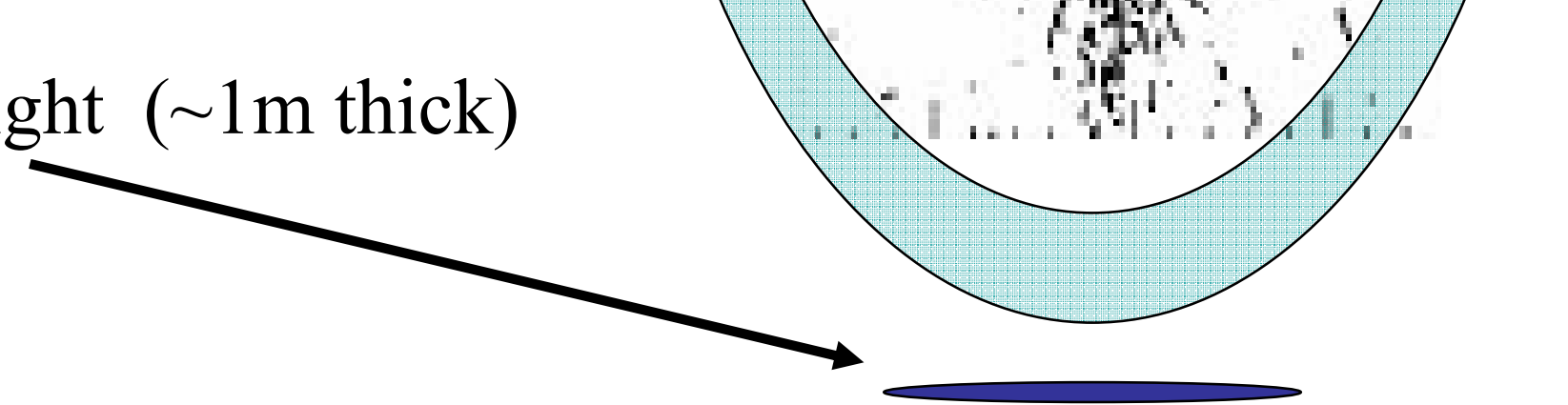




\section{Fluxes and energy thresholds}

- $\mathrm{N}_{2}$ fluorescent yield: $0.02 \mathrm{pe} / \mathrm{GeV}$

- Use Cerenkov trigger to open $\mathrm{N}_{2}$ fluorescent gate for only 10-20 ns, so background is negligible

- This trigger Lowers energy threshold from $10^{18} \mathrm{eV}$ down to $10^{16} \mathrm{eV}$, or lower

- Larger light gathering area ... down to $10^{15} \mathrm{eV}$ ?

Goals would be to (1) discriminate $\gamma$-rays from protons and (2) make better hadronic energy measurements 


\section{Muon dual-readout identification}

The Cerenkov signal from an aligned, nonradiating muon is zero

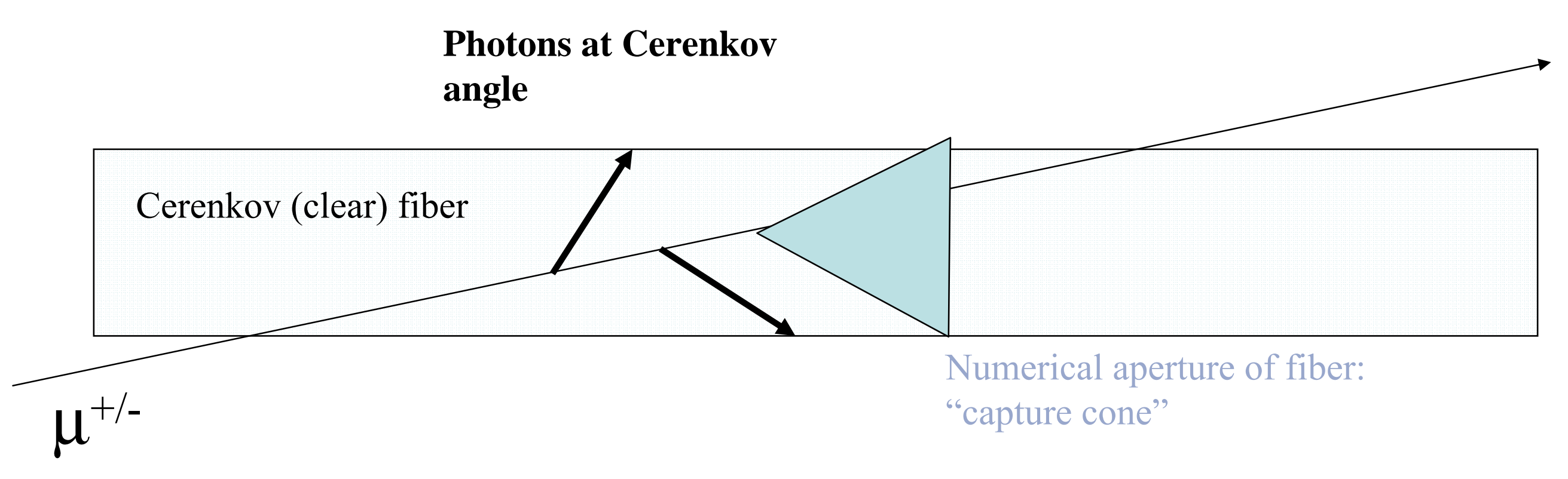

All of the Cerenkov light of an approximately aligned muon falls outside of the numerical aperture of the fiber. 


\section{Therefore, for a single $\mu$}

* $d E / d x$ energy loss yields $\mathrm{S}=d E / d x$ and $\mathrm{C}=0$

* Radiative energy loss inside calorimeter volume yields: $\mathrm{S} \sim \mathrm{C}$
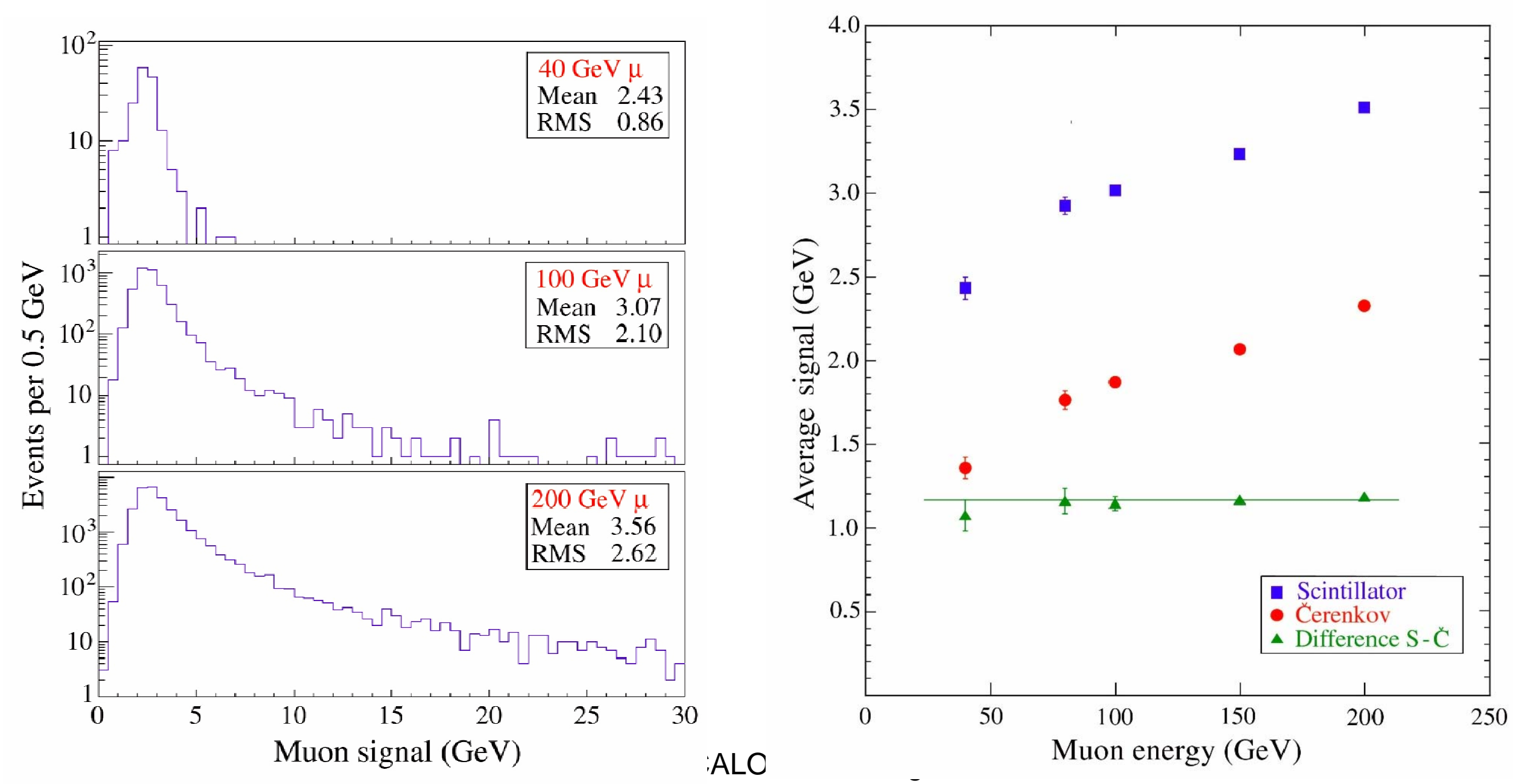


\section{Muons $(40 \mathrm{GeV})$ \& Pions $(20 \mathrm{GeV})$}

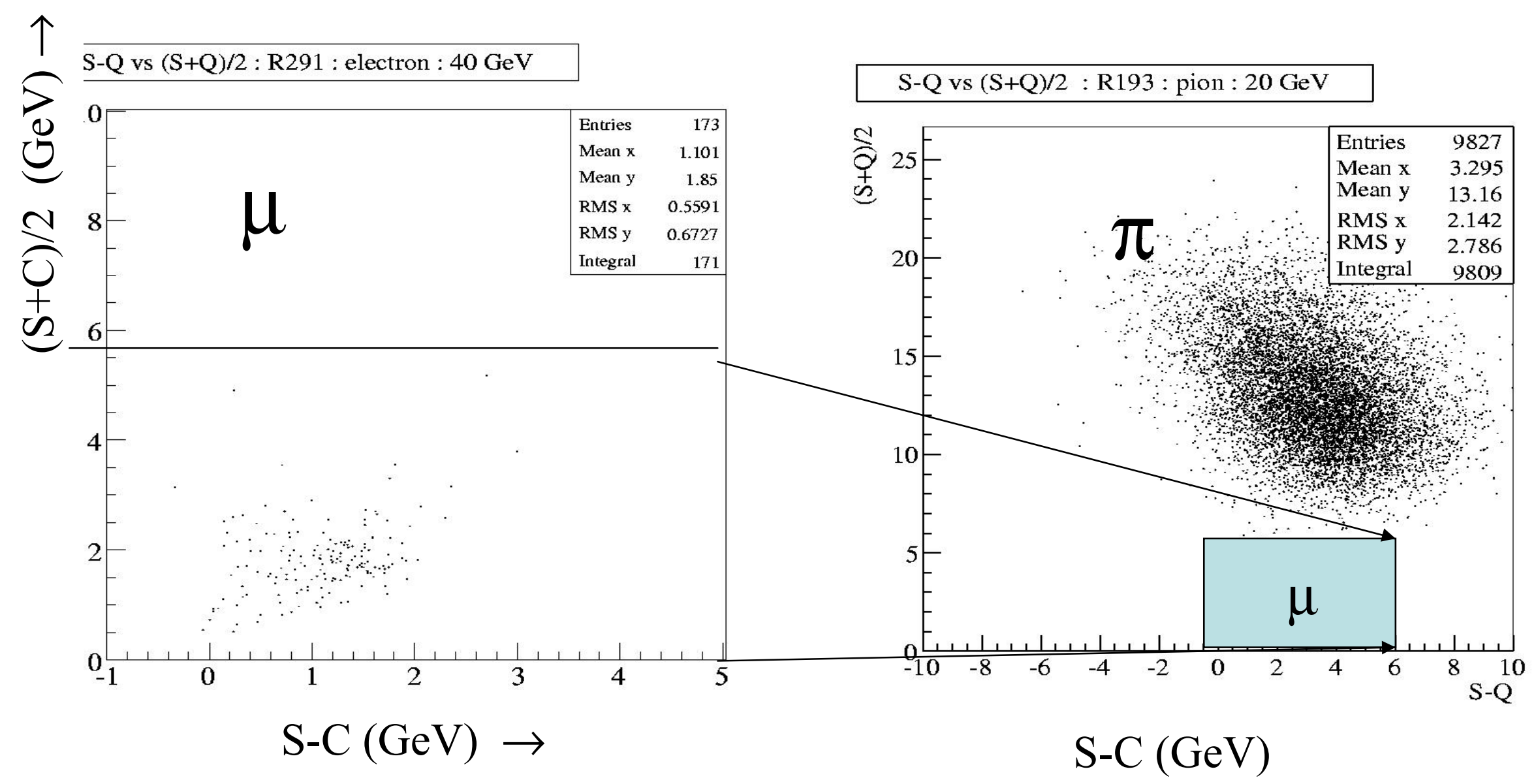

June 2006

J. Hauptman CALOR06 Chicago 


\section{Muons and Pions (80 GeV)}

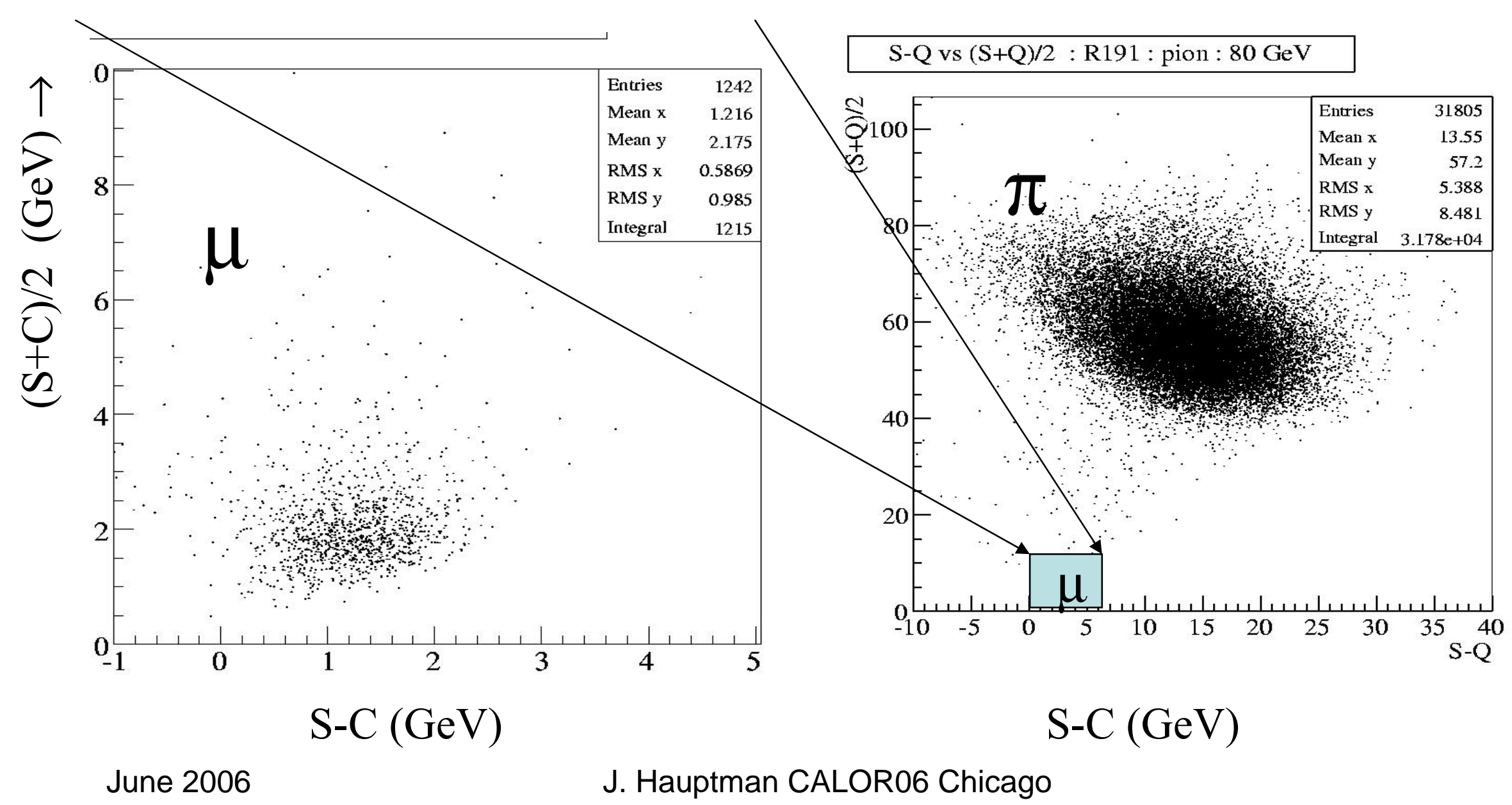




\section{Muons and Pions (200 GeV)}

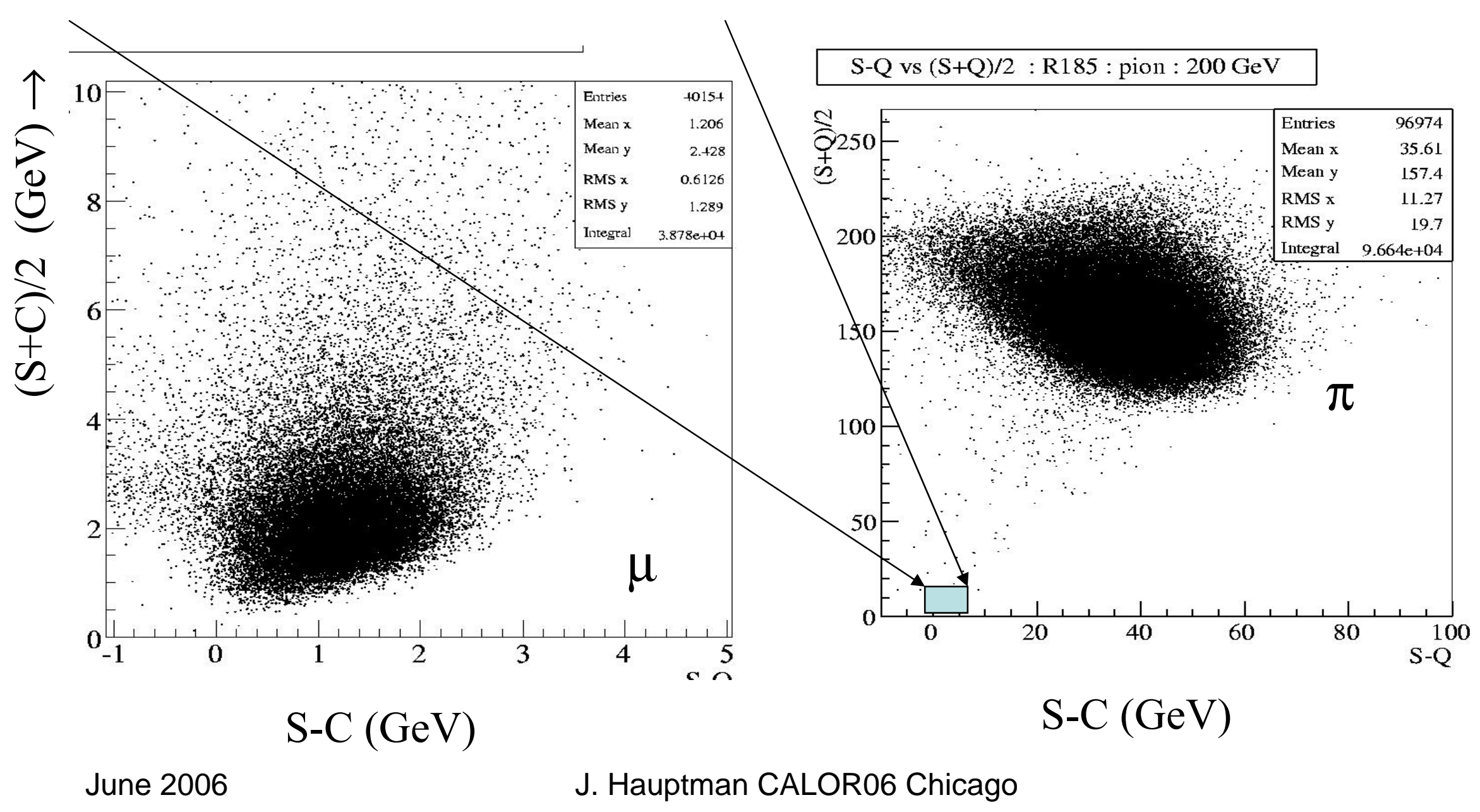




\section{Muons and Pions $(300 \mathrm{GeV})$}

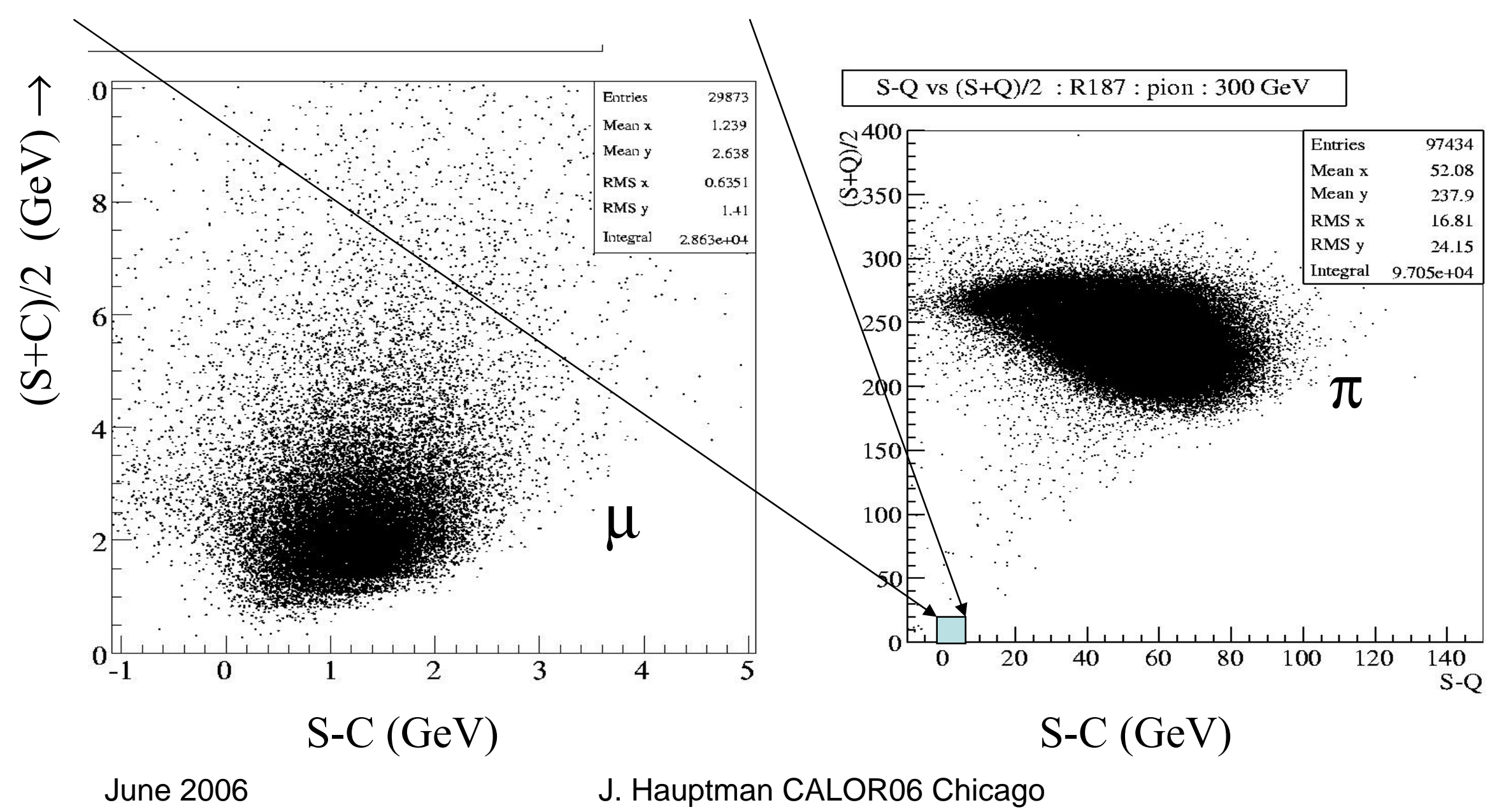




\section{Summary...}

- In a fiber-calorimeter realization, dual readout is easy ... "first shot"

- Likely to be "easy" in an "EM calorimeter" crystal

- Not restricted to $n=2$ ("dual"): why not $n=3$ "triple" readout of scintillation, Cerenkov and neutron signals in hadronic showers.

- Not restricted to calorimeters: any measured quantity whose summed parts fluctuate benefits by those parts being separately measured.

- It is a time to be clever ... there are likely another half dozen ideas not yet conceived. 